Methods Systematic review of quantitative observational studies of an interaction, or effect modification, between preterm birth and SEC. Searches were conducted from five databases between January 2000 and June 2020, and all were identified for dual screening based on title and abstract review. Inclusion criteria was comparison across SEC and gestational age, interaction between the two, or stratification by either, and health or education as outcome. All included studies were citation searched. All studies were narratively synthesised and quality assessed.

Results After searches, 52 studies were identified for full text screening, of which nine met inclusion criteria. Citation searching produced ten more studies. The final sample (19) covered eight countries (all North America and Europe). Forty outcomes were identified, split into four categories; preschool, primary school (5-11), secondary school (11-18), and post-school (18-29). Outcomes for pre-school was development/cognitive, school was cognitive/performance, and postschool was socioeconomic status. Health outcomes were neonatal mortality, ADHD medication prescription, and psychiatric diagnosis or disability post school. Varied measures of socioeconomic status were used as exposure. The majority (16) categorised preterm birth. Of the 18 studies that examined interaction, seven studies found there was a statistically significant interaction between measure of SEC and preterm birth. All interactions found demonstrate that the negative influence of preterm birth was stronger for those in low SEC. Outcomes which provide significant interaction included; cognitive outcome at 18, prescription of ADHD medication in childhood and adolescence, educational attainment at all levels, and psychiatric admissions in 23 to 29 .

Discussion There is some evidence of an interaction between preterm birth and SEC on outcomes for children and young people; the negative effects of preterm birth were exacerbated by low SEC. The remaining evidence suggests the effects accumulate; this is potentially due to underpowered studies. A limitation of the evidence is reporting, some studies only reported whether interaction was significant. This evidence has important policy implications; the potential exacerbation, added to the socioeconomic inequalities in preterm birth, means there is a cohort of children with increased vulnerability, with specific needs that require further investigation.

\section{P60 COMPARING LIFE COURSE AND CURRENT SOCIAL GRADIENTS IN GENERAL HEALTH AT AGE 50: A SIMPLE MEASURE OF INEQUALITY OF OPPORTUNITY FOR HEALTH}

\footnotetext{
${ }^{1,2}$ Christopher Luebker ${ }^{*},{ }^{2}$ Tim Doran, ${ }^{1}$ Richard Cookson. ${ }^{1}$ Centre for Health Economics, University of York, Heslington, UK; ${ }^{2}$ Department of Health Sciences, University of York, Heslington, UK
}

\subsection{6/jech-2021-SSMabstracts. 148}

Background Sophisticated measures of inequality of opportunity for health require the empirically challenging and ethically controversial task of disentangling causal networks between circumstances and choices throughout an individual's life. 'Life course' gradients - bivariate associations between childhood social circumstances and adult health - may provide a simple indicator of societal lifetime inequality of opportunity for health, since there is widespread ethical agreement children cannot be held responsible for their social circumstances. We compare conventional 'current' social gradients in adult health with 'life course' gradients, representing a simple inequality of opportunity metric.

Methods We use data from the UK 1958 National Child Development Study, applying multiple imputation methods to account for attrition. The primary health variable is SF-36 general health score (0-100) measured at age 50, with dichotomised self-assessed general health included as a robustness check. The primary SES variables are income, social class, and education measured at age 50 and age 16. SES variables at age 16 are measured by parental proxy and several alternatives are explored in robustness checks, including parental SES earlier in childhood. Gradients are calculated using the slope and relative indices of inequality (SII, RII) for SF-36 general scores and risk differences and ratios for self-assessed health.

Results Life course income gradients in SF-36 scores are 4.53 and 5.22 for women and men, respectively, using the SII $(\mathrm{p}<0.001)$ and 1.07 and 1.08 for women and men, respectively, using the RII $(\mathrm{p}<0.001)$. Life course gradients in SF-36 scores are also found using mother's education (SII: 11.32/ 10.84 for women/men, $\mathrm{p}<0.001$; RII: 1.19/1.18 for women/ men, $\mathrm{p}<0.001$ ), father's education (SII: 9.97/7.75 for women/ men, $\mathrm{p}<0.001$; RII: 1.16/1.13 for women/men, $\mathrm{p}<0.001)$ and household social class (SII: $11.60 / 7.11$ for women $/ \mathrm{men}$, $\mathrm{p}<0.001$; RII: $1.19 / 1.12$ for women/men, $p<0.001)$. Life course gradients are approximately half the size of current gradients for parental education and women's parental social class and one third the size for parental income and men's parental social class. Results are robust across inequality measures, adult health variables, childhood SES variables, and missing data assumptions.

Discussion Children in the most socioeconomically disadvantaged households at age 16 tend to experience substantially worse general health at age 50 than children in the most advantaged households. This trend is more pronounced when using static SES markers such as education. Lifecourse social gradients provide a simple indicator of inequality of opportunity for health, which are $31-56 \%$ the magnitude of health inequality measured using conventional current social gradients.

\section{P61 REGIONAL INEQUALITIES IN MULTIMORBIDITY WITHIN ENGLAND BETWEEN 2004 AND 2019: A DESCRIPTIVE EPIDEMIOLOGY STUDY USING THE CLINICAL PRACTICE RESEARCH DATALINK}

Anna Head*, Kate Fleming, Chris Kypridemos, Pietà Schofield, Martin O'Flaherty. Public Health, Policy and Systems, University of Liverpool, Liverpool, UK

\subsection{6/jech-2021-SSMabstracts. 149}

Background An estimated 25\% of GP patients within the UK have multimorbidity (two or more chronic conditions), a large proportion of which is attributable to non-communicable diseases, many of them preventable. There are known regional inequalities in health across England, including for chronic diseases. This study aimed to describe regional inequalities in multimorbidity incidence and prevalence.

Methods We selected a random sample of $1 \mathrm{~m}$ adults from the Clinical Practice Research Datalink (CPRD Aurum database) registered at participating GP practices within England between 2004 and 2019. Regions were defined by 2010 Strategic Health Authority boundaries as per the location of the participant's general practice. Participants were linked to 
quintiles of the 2015 Index of Multiple Deprivation (IMD) as a measure of area-level socioeconomic deprivation. We used two measures of multimorbidity: a) basic multimorbidity: two or more chronic conditions; b) complex multimorbidity: at least three chronic conditions affecting at least three body systems. A list of 211 chronic conditions of interest, including long-term mental health conditions and chronic infections, was agreed by a multidisciplinary team. Using standard formulae, we calculated crude and age-sex standardised multimorbidity prevalence and incidence by geographical region. We used quasi-Poisson regression models to calculate risk ratios adjusted for year, sex, age, region, and IMD quintile. Analyses were conducted using R v4.0.4.

Results Our final sample consisted of 989,421 adults: 48.7\% male, with median age of 46 years (inter-quartile range 3362). The overall crude prevalence of multimorbidity in England was $43.7 \%$ for basic, and $25.2 \%$ for complex multimorbidity over the 16-year study period. London had the lowest crude prevalence of both multimorbidity types (basic: $35.4 \%$; complex: 18.3\%), whilst the North East had the highest (basic: 48.6\%; complex: 29.6\%). In age-sex standardised results, prevalence was still highest in the North East, with London and the South East having the lowest prevalence. Similar regional inequalities were found in the incidence of both multimorbidity types. Compared to London, the North East had higher multimorbidity prevalence in risk ratios adjusted for socioeconomic deprivation and demographic factors (basic multimorbidity: 1.18 (95\% confidence interval 1.16, 1.19); complex multimorbidity: 1.26 (95\% confidence interval 1.24, 1.29). Conclusion There are regional inequalities in multimorbidity within England with higher burden in the North, compared to London and the South. These inequalities remained after adjusting for age and socioeconomic deprivation. Strategies aimed at addressing the social determinants of health are needed to reduce future burden on health and social care systems, particularly in the North of England.

\section{P62 EDUCATIONAL INEQUALITIES IN STATIN TREATMENT: CROSS-SECTIONAL ANALYSIS OF UK BIOBANK}

\begin{abstract}
1,2Alice Carter* , 3,4,5,6,7 Dipender Gill, ${ }^{2,8}$ Richard Morris, ${ }^{1,2,9}$ George Davey Smith, ${ }^{2,9}$ Amy Taylor, ${ }^{1,2,1}$ Neil Davies, ${ }^{1,2}$ Laura Howe. 'MRC Integrative Epidemiology Unit, University of Bristol, Bristol, UK; ${ }^{2}$ Population Health Sciences, University of Bristol, Bristol, UK; ${ }^{3}$ Department of Epidemiology and Biostatistics, Imperial College London, London, UK; ${ }^{4}$ Centre for Pharmacology and Therapeutics, Imperial College London, London, UK; ${ }^{5}$ Clinical Pharmacology and Therapeutics Section, St George's, University of London, London, UK; ${ }^{6}$ Clinical Pharmacology Group, St George's, University of London, London, UK; ${ }^{7}$ Centre for Academic Primary Care, University of Bristol, Bristol, UK; ${ }^{8}$ NIHR Bristol Biomedical Research Centre, University of Bristol, Bristol, UK; ${ }^{9}$ K.G. Jebsen Center for Genetic Epidemiology, Norwegian University of Science and Technology, Trondheim, Norway
\end{abstract}

10.1136/jech-2021-SSMabstracts. 150

Background Despite reductions in the rates of cardiovascular disease in high income countries, individuals who are the most socioeconomically deprived remain at the highest risk of disease. Although intermediate lifestyle and behavioural risk factors explain some of this, much of the effect remains unexplained. It is not known whether differences in risk adjusted use of statins between educational groups may contribute to these inequalities.

Methods Using data from a large prospective cohort study, UK Biobank, we calculated a QRISK3 cardiovascular risk score for 472097 eligible participants with complete data on self- reported educational attainment and statin use (55\% female; mean age, 56). We used logistic regression to explore the association between i) QRISK3 score and ii) educational attainment on self-report statin use. We then stratified the association between QRISK3 score, and statin use by educational attainment to test for interactions. We then replicated analyses using QRISK or QRISK2 scores recorded in primary care data and statin prescriptions recorded in primary care prescription records.

Results There was evidence of an interaction between QRISK3 scores and education. For an equivalent QRISK3 score, more educated individuals were more likely to report taking statins. In women with 7 years of schooling, a one unit increase in QRISK3 score was associated with a 7\% higher odds of statin use (odds ratio (OR) 1.07, 95\% CI 1.07, 1.07). In women with 20 years of schooling, a one unit increase in QRISK3 score was associated with an $14 \%$ higher odds of statin use (OR 1.14, 95\% CI 1.14, 1.15). Comparable ORs in men were $1.04(95 \%$ CI $1.04,1.05)$ for 7 years of schooling and 1.08 (95\% CI 1.08, 1.08) for 20 years of schooling. These inequalities were also present in analyses using primary care data.

Conclusion For the same level of cardiovascular risk, individuals with lower educational attainment are less likely to receive statins, likely contributing to cardiovascular inequalities. The mechanisms leading to these differences are unknown, but both health seeking behaviours and clinical factors may contribute.

\section{P63 USING DATA ON FATHERS/PARTNERS TO STUDY PRENATAL PARENTAL EXPOSURES AND CHILD HEALTH: CHALLENGES INTRODUCED BY MISSING DATA AND SELECTION BIAS}

Kayleigh Easey*, Gemma Sharp. MRC Integrative Epidemiology Unit, University of Bristol, Bristol, UK

\subsection{6/jech-2021-SSMabstracts. 151}

Background There are challenges in studying the effects of partner exposures around pregnancy on child health. We explored potential sources of bias in the effects of parental prenatal health behaviours on child health, to describe and quantify some of these challenges, as well as suggest ways in which they might be mitigated.

Methods First, we characterised the availability of data on partner and mother health behaviours in the prenatal period from three UK cohort studies: the Avon Longitudinal Study of Parents and Children (ALSPAC), Born in Bradford (BiB), and the Millennium Cohort Study (MCS). Second, we assessed the potential for sample selection in these cohorts by comparing characteristics of families where the partner did and did not participate. Third, using parental smoking during pregnancy and child birthweight as an example, we ran simulation studies of several DAGs to explore the extent that missing partner data and selection can affect estimates. We then explored the 'real life' impact of partner sample selection on estimates of maternal effect.

Results In all cohorts, data on partner prenatal health behaviours was less detailed and collected less frequently than maternal prenatal health behaviours. Partners participated in ALSPAC and MCS for the majority of pregnancies. Of 14,472 pregnancies in ALSPAC, and 18,241 pregnancies in MCS, 